\title{
Políticas de inclusión educativa en la universidad pública uruguaya
}

\author{
Andrea Jimena Viera Gómez \\ Facultad de Psicología de la Universidad de la República - Montevideo - Uruguay \\ Yliana Zeballos Fernández \\ Facultad de Psicología de la Universidad de la República - Montevideo - Uruguay
}

\begin{abstract}
Resumen
Este artículo tiene por finalidad presentar resultados del relevamiento documental y bibliográfico sobre políticas de inclusión educativa de personas con discapacidad en la Educación Superior pública uruguaya, específicamente en la Universidad de la República. Se realizó un estudio documental a partir del relevamiento y análisis de la legislación vinculada a la inclusión de personas con discapacidad en la Educación Superior pública uruguaya. Se recopiló documentación oficial correspondiente a la normativa de Estado así como otros documentos universitarios. Se integran en el análisis otros artículos y textos académicos que permiten ampliar la discusión en relación con esta temática. De los resultados obtenidos a través de esta primera aproximación, observamos la limitada producción de documentos que abordan la problemática de la inclusión educativa de las personas en situación de discapacidad en la Educación Superior pública en Uruguay.
\end{abstract}

Palabras clave: Política educacional; personas con deficiencia; educación superior.

\section{Educational inclusion policies at uruguayan public university}

\begin{abstract}
This article aims to present results of the documentary and bibliographic survey on policies of educational inclusion of persons with disabilities in Uruguayan public higher education, specifically at the Universidad de la República. A documentary study was carried out based on the survey and analysis of the legislation related to the inclusion of persons with disabilities in Uruguayan public higher education. Official documentation corresponding to State regulations and other university documents were compiled. Other articles and academic texts are integrated in the analysis that allow to broaden the discussion in relation to this subject. From the results obtained through this first approximation, we observe the limited production of documents that address the issue of the educational inclusion of people with disabilities in public higher education in Uruguay.
\end{abstract}

Keywords: Educational policy; people with disabilities; higher education.

\section{Políticas de inclusão educativa na universidade pública uruguaia}

\section{Resumo}

Este artigo tem por objetivo apresentar resultados do levantamento documental e bibliográfico sobre políticas de inclusão educativa de pessoas com deficiência na Educação Superior pública uruguaia, especificamente na Universidade de la República. Realizou-se um estudo documental a partir do levantamento e análise da legislação vinculada à inclusão de pessoas com deficiência na Educação Superior pública uruguaia. Coletouse documentação oficial correspondente à normativa de Estado assim como outros documentos universitários. Integraram-se na análise outros artigos e textos acadêmicos que permitem ampliar a discussão em relação com esta temática. Dos resultados obtidos por meio desta primeira aproximação, observamos a limitada produção de documentos que abordam a problemática da inclusão educativa das pessoas em situação de deficiência na Educação Superior pública no Uruguai.

Palavras-chave: Política educacional; pessoas com deficiência; ensino superior. 


\section{Introducción}

En las últimas décadas Uruguay presenta avances en la actualización de la política nacional con relación a la protección y garantía del derecho a la educación de las personas en situación de discapacidad en el sistema educativo.

El sistema educativo uruguayo se rige en la actualidad por la Ley General de Educación N 18.437 del año 2008 (2008). La misma reafirma los principios de universalidad y la obligatoriedad de la educación para todos los habitantes de la República, desde el nivel inicial a la enseñanza media básica y superior. Asimismo en el texto de la Ley se expresa el principio de Diversidad e Inclusión Educativa. Artículo $8^{\circ}$ : El Estado asegurará los derechos de aquellos colectivos minoritarios o en especial situación de vulnerabilidad, con el fin de asegurar la igualdad de oportunidades en el pleno ejercicio del derecho a la educación y su efectiva inclusión social. Se pone énfasis en el carácter participativo en los procesos de enseñanza - aprendizaje del sujeto de la educación, como sujeto activo, de los agentes de la educación así como de las familias y la comunidad toda.

En este sentido se han venido implementando diversas políticas educativas inclusivas tanto a nivel de la Administración Nacional de Educación Pública (ANEP), de la cual depende el Consejo de Educación Inicial y Primaria (CEIP), el Consejo de Educación Secundaria (CES), el Consejo de Educación Técnico Profesional (CETP) y la Dirección de Formación y Perfeccionamiento docente (CFE); así como también a nivel de la educación superior representada en el sistema educativo público por la Universidad de la República (UDELAR).

En cuanto a las políticas de inclusión educativa en la Universidad de la República se destaca la creación de la Red Temática de Discapacidad en el año 2008, la cual tiene como propósito la formación, socialización y actualización acerca de la temática (Universidad de la República, 2008).

La Red si bien posee un carácter académico e investigativo, en sus estatutos deja explícito un lineamiento general en relación a los beneficios de trabajar en torno a la diversidad humana para lograr un cambio en el diseño de políticas focalizadas y proyectarse hacia una educación inclusiva como política generalizada en la educación superior (Universidad de la República, 2008).

Cabe destacar que Uruguay ha venido avanzando desde 2005 con la creación del Programa Nacional de Discapacidad dependiente del Ministerio de Desarrollo Social (MIDES) con el objetivo de delinear una política nacional actualizada y en consonancia con los principios de la Convención de las Personas con Discapacidad (2006) materializados en la Ley de Protección Integral de Personas con Discapacidad No 18.651 del año 2010 y la Ley General de Educación № 18.437 de 2008.

En este sentido encontramos un informe del rectorado de Rodrigo Arocena del año 2012 con avances realizados en la Universidad de la República a nivel central y a nivel de los servicios universitarios en cuanto a la accesibilidad y la inclusión educativa.
El informe da cuenta de las acciones realizadas y sistematiza la información obtenida a partir de la aplicación, por parte de la Red Temática, de un formulario auto-administrado a funcionarios docentes y no docentes con el fin de relevar la situación de accesibilidad y la inclusión de personas con discapacidad en la Universidad.

\section{Objetivo}

Este artículo tiene por finalidad presentar resultados del relevamiento documental y bibliográfico sobre políticas de inclusión educativa de personas con discapacidad en la Educación Superior pública uruguaya, específicamente en la Universidad de la República.

\section{Método}

Se realizó un relevamiento bibliográfico que contempló la legislación uruguaya referida a la educación en general y a la Educación Superior, en particular. En la misma se relevó el contenido relativo a la inclusión de personas con discapacidad en la Educación Superior uruguaya.

Asimismo, se relevaron fuentes secundarias de información a través de diferentes bases de datos (SCielo, Redalyc, Latindex, y Timbó) ya que la Universidad de la República cuenta desde hace relativamente poco tiempo con una base de datos propia (BiUR $\left.{ }^{1}\right)$. Las palabras clave empleadas en la búsqueda tanto de las publicaciones académicas científicas y de otros documentos públicos del país fueron: "Inclusión Educativa”, "Educación Superior", "Discapacidad" y, "Accesibilidad".

Conceptualización de la inclusión educativa

Consideramos importante plantear desde donde abordamos el tema de la inclusión educativa en la Educación Superior pública de Uruguay.

El término inclusión educativa es un término polisémico que ha tenido una evolución en cuanto a sus diferentes significados en diferentes momentos históricos. Lo que ha llevado a que exista una gran confusión acerca de lo que significa de hecho "inclusión" o "educación inclusiva"

Según Ainscow, citado por Echeita (2006), es de utilidad considerar los siguientes elementos que configuran una educación más inclusiva. En primer término la idea de que la inclusión es un proceso, es decir poder concebirla como una tarea interminable de búsqueda de mejores formas de responder a la diversidad de estudiantes, "es sobre cómo aprender a vivir con la diferencia y aprender como aprender desde la diferencia" (2006, p. 14).

La inclusión tiene que ver con la tarea de identificar y remover barreras, este concepto de barreras es muy potente porque es un concepto relacional, saca el foco de las dificultades centradas en la persona e involucra el contexto vincular. Para realizar esta tarea Ainscow plantea que es

1 En esta se encuentran unificados los catálogos de todas las bibliotecas de la Universidad de la República. 
necesario recopilar, ordenar y evaluar información de una amplia variedad de fuentes para planificar mejoras en las políticas y en las prácticas.

Asimismo el autor afirma que la inclusión tiene que ver con la presencia, la participación y el aprendizaje de los estudiantes, garantizar esto nos asegura la continuidad educativa de todos.

Por último, la inclusión supone poner un énfasis particular en los grupos de estudiantes que pueden estar en riesgo de marginación, exclusión o fracaso escolar por diversos motivos, uno de ellos que es en el que estamos poniendo foco en esta comunicación es la situación de los jóvenes uruguayos con discapacidad en la Educación Superior.

En el informe descriptivo de análisis de accesibilidad en Educación Superior para personas con discapacidad de ESVI-AL (2013, p. 16) se hace un esbozo interesante y muy esclarecedor de las barreras y obstáculos que enfrentan las personas con discapacidad al ingresar a la Educación Superior.

- Barreras sociales, actitudinales y de comunicación, se las define como el hecho social que impide o dificulta la interacción entre las personas.

- Barreras arquitectónicas y espaciales, incluye el desplazamiento desde el hogar a la Universidad, medios de transporte, recursos económicos y luego el acceso al edificio mismo. En algunas universidades se han realizado acciones para la eliminación de barreras de accesibilidad.

- Barreras técnicas y humanas, relacionadas con la disponibilidad de los recursos humanos, físicos, técnicos y económicos. Esto incluye la formación de los profesores que se manifiesta en un modelo pedagógico estandarizado y poco pertinente para la enseñanza de personas con discapacidad.

- Barreras económicas y otros apoyos, se refieren a los recursos para incorporar ayudas o adaptaciones de acuerdo a sus necesidades educativas, así como el lugar geográfico de residencia y la falta de conectividad.

Considerando este marco de referencia conceptual es que nos proponemos pensar sobre la situación actual de acceso, permanencia y egreso de las personas con discapacidad en la Universidad de la República que nos permita delimitar un territorio, marcar un horizonte de actuación y de reflexión acerca de hacia dónde direccionar las políticas de educación inclusiva en la Educación Superior en Uruguay.

Reseña de la inclusión educativa en Uruguay

En el escenario uruguayo el principal antecedente de la inclusión educativa de personas con discapacidad es el Plan de Integración que se pone en práctica en el año 1987. Este Plan tenía como cometido la integración de los alumnos de la Educación Especial a la escuela común y el ingreso directo a las aulas de educación inicial de aquellos alumnos con discapacidad que iniciaban su escolaridad (Banco
Mundial, 2004). Si bien el Plan de Integración deja de ser financiado en 1999, la modalidad de integración de alumnos en la actualidad sigue incambiada (Viera \& Zeballos, 2014).

Frente a la confirmación de una desigualdad educativa creciente, y en sintonía con iniciativas similares desarrolladas en la región, durante el período 2001-2004 Uruguay llevó a cabo proyectos colaborativos con la Organización de Estados Americanos. A partir de aquí se desarrolla el Fondo de Inclusión Escolar enmarcado en el Proyecto de Mejoramiento de la Calidad Educativa Primaria con recursos de la Administración Nacional de Educación Pública y del Banco Mundial (Banco Mundial, 2004). Este Fondo de Inclusión Escolar se orientó a apoyar la transformación de las escuelas especiales en Centros de Recursos para brindar apoyo a las escuelas comunes y a su comunidad en el proceso de inclusión educativa de personas con discapacidad. Esto es evaluado en los documentos oficiales (Banco Mundial, 2004) como un nuevo hito hacia una orientación inclusiva y pro-diversidad en la gestión de políticas educativas del país (Viera \& Zeballos, 2014).

Dentro del marco legal actual de la educación uruguaya se plantea la flexibilización curricular y metodológica para atender la diversidad del alumnado destacándose la consideración de la inclusión educativa de las "personas con discapacidades":

La educación formal contemplará aquellas particularidades, de carácter permanente o temporal, personal o contextual, a través de diferentes modalidades, entendidas como opciones organizativas o metodológicas, con el propósito de garantizar la igualdad en el ejercicio del derecho a la educación.

Se tendrá especial consideración a la educación en el medio rural, la educación de personas jóvenes y adultas y la educación de personas con discapacidades, promoviéndose la inclusión de éstas en los ámbitos de la educación formal, según las posibilidades de cada una, brindándoles los apoyos necesarios (Ley $n^{\circ} 18.437,2008$, Cap. II, Art. 33).

\section{La inclusión educativa en el escenario de la Educación Superior pública uruguaya}

Tal como lo advertíamos al comienzo, la Universidad de la República es la única institución educativa pública encargada de la Educación Superior en Uruguay. Nuestra Universidad se sitúa en una concepción de Universidad latinoamericana, la misma encontró fundamento en el Movimiento de Reforma universitaria que tiene su origen en Córdoba (1918). Este movimiento tuvo un gran impacto en la Universidad y en su contexto social y cultural, convirtiéndose en impulsor de una mayor democratización en contraposición a una Universidad de élites.

En ese sentido se orientó a abrir sus puertas a sectores sociales más amplios, facilitando el acceso y permanencia de estudiantes a través de la asistencia libre. Impulsó la democratización del gobierno universitario, dando participación a estudiantes y egresados. Propulsó, además, una re- 
lación más estrecha con la sociedad, proponiendo las bases para el desarrollo de la extensión universitaria

Si bien nuestra Universidad ya contaba con una tradición en autonomía y participación estudiantil en su gobierno, no se puede desconocer que el movimiento estudiantil reforzó estos afanes reformistas.

Sin embargo, no fue hasta avanzada la década del '50 que los principios fundamentales de este movimiento se vieron incorporados a los estatutos de la Universidad, al igual que en gran parte de las universidades latinoamericanas. La elaboración de nuestra ley orgánica dará forma a estas distintas influencias (Bralich, 1994).

La conquista de la autonomía del poder político fue tardía y se consagró en la Ley Orgánica de 1958 que promueve el cogobierno de docentes, estudiantes y egresados.

Cabe señalar que Uruguay es uno de los pocos países de la región que mantiene una estructura cogobernada hasta el presente. En este marco de transformaciones académicas y políticas que ha tenido nuestra Universidad es que nos encontramos en la actualidad con un proceso de reforma que busca acompasar los nuevos desafíos que emanan de un contexto social paradójicamente "globalizado", a la vez que fragmentado.

El inicio de la Segunda Reforma puede ubicarse en el año 2007 cuando el Consejo Directivo Central de la UdelaR resuelve: "La Universidad de la República se propone impulsar una Reforma profunda, que sintonice con una transformación general de la enseñanza nacional" (Universidad de la República, 2007, p.13). Y destaca como objetivo:

La transformación del conjunto de la educación uruguaya debe estar orientada por el propósito de avanzar hacia la generalización de la enseñanza avanzada, de calidad y conectada con el trabajo a lo largo de toda la vida activa. Para la Universidad, colaborar a ello supone grandes transformaciones, «hacia adentro» y «hacia afuera». La Reforma de Córdoba gestó el ideal latinoamericano de Universidad que conjuga enseñanza, investigación y extensión desde la autonomía, el cogobierno y el compromiso social. La Segunda Reforma Universitaria debe reafirmar ese ideal a través de un conjunto de cambios que apunten hacia la meta fundamental (Universidad de la República, 2007, pp.13-14).

Tres ideas centrales orientan esta Reforma:

(1) Revitalizar el ideal latinoamericano de Universidad comprometida con la sociedad, que combine enseñanza, extensión e investigación a alto nivel, al servicio de la sociedad y con participación activa del estudiantado.

(2) Generalización de la enseñanza avanzada, permanente, conectada con el trabajo a lo largo de la vida entera.

(3) Universidad para el desarrollo. En América Latina las grandes universidades públicas son las grandes generadoras de conocimiento, eso nos hace titulares de un deber inmenso, tenemos que poner ese conocimiento al servicio de la mejora de las condiciones de vida y del desarrollo del país (Arocena, 2008).

En el marco de esta concepción universitaria, la investigación, la docencia, y la extensión deben estar estrechamente ligadas con el fin de producir, elaborar, y transmitir conocimientos y producciones culturales en un movimiento de real participación en la comunidad. Por tal razón, las líneas de investigación deben estar apoyadas en las necesidades y demandas sociales detectadas.

Con el propósito de promover la interdisciplina el Consejo Directivo Central crea en 2007 el Espacio Interdisciplinario como servicio destinado a albergar y promover las actividades interdisciplinarias en la Universidad de la República. En este marco en el año 2008 se crea la Red Temática de Discapacidad (RETEDIS), su fundamentación está dada por un campo conceptual y aplicación de conocimientos, al mismo tiempo que se ha ido jerarquizando como una cuestión de derechos humanos.

El objetivo de la Red Temática es el de fomentar la inclusión social y educativa de las personas con discapacidad, trabajando en el desarrollo de estrategias que aseguren un mayor y mejor acceso a la educación y el trabajo de las personas que presentan esta situación en forma permanente o transitoria.

La Red se propone establecer el intercambio sistemático de conocimiento y experiencia producida en el campo de la discapacidad de las unidades académicas que la integran. Se encarga de realizar actividades de sensibilización y difusión de aspectos vinculados a la accesibilidad y la inclusión de las personas con discapacidad en ámbitos intra-universitarios y extrauniversitarios. Asimismo, colabora en el diseño de programas que favorezcan la inclusión educativa y, en la realización de encuentros sistemáticos con personas y asociaciones de personas con discapacidad. Por otra parte, establece vínculos con universidades extranjeras e integra la Red Interuniversitaria Latinoamericana y del Caribe sobre Discapacidad y Derechos Humanos.

En cuanto a la obtención y sistematización de información respecto a estudiantes con discapacidad, la Universidad de la República no maneja registro de estos datos, sin embargo es interesante conocer la postura de los estudiantes, quienes han manifestado su descontento frente al desconocimiento de la población con discapacidad en la Universidad.

Los ideales de justicia social y democracia que constituyen la esencia del ideal latinoamericano de Universidad están en consonancia con la forma de concebir la inclusión.

En este sentido cabe señalar que la temática de la discapacidad ha llegado a la Universidad hace relativamente poco tiempo de ahí la importancia del trabajo de la Red temática en la formación y sensibilización de toda la comunidad educativa universitaria.

Avances y limitaciones de las políticas de inclusión educativa de personas en situación de discapacidad en la Educación Superior pública uruguaya 
En la actualidad la inclusión de personas con discapacidad en la Educación Superior pública uruguaya se encuentra contemplada en dos leyes nacionales: la Ley General de Educación N¹8.437 y la Ley de Protección Integral de Personas con Discapacidad N`18.651.

No obstante, también se pueden incluir en este relevamiento el documento de la creación de la Red Temática sobre Discapacidad de la Universidad de la República: la Resolución Nº6/2008 del Consejo Directivo Central (Universidad de la República, 2008).

Por otra parte, dentro de los documentos universitarios encontramos el texto de Lemez (2005), Tejeda (2012) y Farías y Viera (2013).

También incluimos el trabajo que realiza Fernández (2009) sobre la segmentación social y académica en Uruguay y analiza los factores que favorecen el acceso a la Educación Superior.

Se trata de un texto que si bien no plantea el tema de la inclusión educativa de las personas con discapacidad, hace referencia a la inadecuación de los planes de estudio universitarios al perfil socio económico de los estudiantes que acceden a la Educación Superior. Y destaca la ausencia de políticas sociales focalizadas en los grupos con menores recursos económicos.

En tal sentido señala lo siguiente: "Cualquier política que tenga por objetivo democratizar el acceso a la Educación Superior debería tener en cuenta el perfil de los jóvenes a quienes está dirigida aquella expansión, para luego ajustar estrategias acorde a este perfil" (Fernández, 2009, p. 24).

En acuerdo con lo planteado por Fernández también debería agregarse en el análisis del perfil de ingreso a la Universidad a los estudiantes con discapacidad. Asimismo, señala que una política universitaria que busque democratizar el acceso de los jóvenes provenientes de contextos desfavorables permitirá incrementar su capital cultural y también mejorará las perspectivas de movilidad social intergeneracional.

Por su parte, Lemez (2005) propone reflexionar sobre la situación de las personas con discapacidad en el Uruguay y su participación en el espacio de la Educación Superior. El artículo analiza las posibles causas en las restricciones en el acceso, la permanencia y el egreso de las personas con discapacidad en la Educación Superior y se encarga de subrayar la brecha existente entre la evidencia de los datos estadísticos y el marco legal vigente que establece obligaciones en tal sentido. No obstante, el escrito carece de información concreta respecto a la inclusión de las personas con discapacidad en la Educación Superior, refiriéndose más a datos estadísticos de discapacidad en el país, tales como: distribución de edades, de sexo, prevalencia, ubicación geográfica y matrícula universitaria.

Asimismo, permite situar los antecedentes históricos respecto a la normativa de Estado, lo que en su momento demostró en Uruguay una política poco concreta e imprecisa en términos de "integración" y de la relación entre las políticas educativas y las políticas sociales. Este aspecto ya ha sido reseñado por varios autores (Mancebo \& Goyeneche,
2010; Viera \& Zeballos, 2014; Meresman, 2012) cuando se plantea una ausencia de la políticas de inclusión educativa en general y de las personas con discapacidad, en particular.

Sin embargo, como señala Martinis (2016), en la última década, la noción de inclusión educativa ha cobrado mayor presencia en la formulación de las políticas educativas en Uruguay. Lo cual estaría evidenciando la emergencia de un discurso pedagógico desde la política educativa.

Tejeda (2012) en su tesis de Maestría examina la situación de las políticas de Educación Superior y discapacidad en tres países del Cono Sur: Argentina, Chile y Uruguay. El estudio concluye que en Uruguay la política nacional y sectorial confluyen en un enfoque social que contempla a los grupos vulnerables de la sociedad dentro de los que se integra a las personas con discapacidad. Subraya que la política nacional se inclina hacia la mejora de la educación más que a garantizar el derecho a la educación. Por otra parte, señala que a nivel universitario, el principal mecanismo de acción frente a la inclusión de personas con discapacidad ha sido sensibilizar y brindar información.

Farías y Viera (2013) presentan algunos resultados de una encuesta realizada a diferentes Facultades que integran la Universidad de la República. Las autoras señalan que:

La discapacidad parece seguir invisibilizada en la Universidad de la República a pesar de los diferentes esfuerzos de sensibilización al respecto. Es así que en gran medida el apoyo a la PCD (personas con discapacidad), muchas veces queda librado a la «buena voluntad» de compañeros y docentes, y sujeto al sentido común (p. 269).

Asimismo, señalan que las políticas desarrolladas por los Servicios (Facultades) en relación con la inclusión educativa se constriñen en su mayoría a la accesibilidad física, en tanto, los aspectos didácticos y pedagógicos apenas son mencionados.

El país ha avanzado en la existencia de un marco jurídico expresado fundamentalmente en la Ley General de Educación No 18.437 (2008) y en la Ley de Protección de las personas con discapacidad No 18.651 (2010).

En cuanto a la Ley General de Educación No18.437 (2008) sancionada en diciembre de 2008 garantiza el derecho a la educación para todos los habitantes de la República sin discriminación de ningún tipo lo que supone el acceso y permanencia en todos los niveles del sistema educativo nacional con todos los apoyos necesarios.

En el artículo 40 subraya:

la equiparación de oportunidades para las personas con discapacidad, desde la educación inicial en adelante, determina que su integración a las aulas comunes se organice sobre la base del reconocimiento de la diversidad como factor educativo, de forma que apunte al objetivo de una educación para todos, posibilitando y profundizando el proceso de plena inclusión en la comunidad. Para garantizar dicha inclusión se asegurará la flexibilización curricular, 
de los mecanismos de evaluación y la accesibilidad física y comunicacional (Ley General de Educación № 18.437, 2008).

Y en el artículo 80 si bien hace referencia a la educación superior pública se destaca que la misma es aplicable a la Universidad de la República en cuanto sea conciliable con la Ley Orgánica de ésta: "La Universidad de la República se regirá por la Ley $N^{0} 12.549$, de 16 de octubre de 1958. El Instituto Universitario de Educación y los Institutos de Educación Terciaria se regirán por las disposiciones de la presente ley".

En cuanto a la Ley de Protección Integral de Personas con Discapacidad N¹8.651 (2010) en su artículo 44 expresa:

\footnotetext{
El Ministerio de Educación y Cultura en coordinación con la Administración Nacional de Educación Pública, la Universidad de la República, entidades educativas terciarias y universitarias privadas, en todos los programas y niveles de capacitación profesional, incluidas las carreras de educación terciaria y universitarias, promoverá la inclusión en los temarios de los cursos regulares, la información, la formación y el estudio de la discapacidad en relación a la materia de que se trate y la importancia de la habilitación y rehabilitación, así como la necesidad de la prevención (Ley de Protección Integral de Personas con Discapacidad $\left.\mathrm{N}^{\circ} 18.651,2010\right)$.
}

Desde este marco jurídico que pretende garantizar el derecho a la educación de todos los habitantes del país incluidas las personas en situación de discapacidad, se han venido desarrollando un abanico de acciones educativas inclusivas tanto en la Administración Nacional de Educación Pública como en la Universidad de la República.

No obstante, la escasa información al respecto, deja en evidencia que la Universidad de la República no dispone de normativas, ordenanzas o disposiciones escritas, vinculadas específicamente a la temática de la inclusión educativa de personas con discapacidad.

\section{Consideraciones finales}

El reconocimiento del derecho a la educación como derecho humano fundamental inalienable, y por ende la necesidad de la universalización de la misma, no sólo supone que todos los sujetos puedan acceder a la educación sino permanecer y aprender.

En Uruguay, la universalización de la educación inicial y de la educación media básica ha sido el desafío de los últimos tiempos y esto ha implicado una transformación del sistema educativo en pro de atender a todos los niños y niñas y los jóvenes del país.

Esto ha implicado procesos de transformación de la Educación Especial y la necesaria integración entre los distintos niveles y subsistemas de enseñanza. Como con- secuencia de lo anterior, ha planteado el desafío de unificar criterios para volver más fluido y eficiente el manejo de la información entre los diferentes subsistemas. Para ello resulta fundamental el trabajo intersectorial e interinstitucional así como la posibilidad de garantizar que las trayectorias educativas de los estudiantes sean continuas, completas y protegidas.

Como señala Fernández (2009) la Universidad no ha quedado por fuera de las transformaciones de la democratización de la educación en Uruguay.

No obstante, el país cuenta con escasa información respecto a la temática de discapacidad y Educación Superior. Como se refiere anteriormente, pocos estudios dan cuenta de la situación de las personas con discapacidad en este subsistema.

Si bien en las últimas décadas Uruguay presenta avances en la actualización de la política nacional con relación a la protección y garantía del derecho a la educación de las personas en situación de discapacidad en el sistema educativo, la orientación de la normativa uruguaya posee un enfoque social. Es decir, plantea una postura política hacia la mejora de los grupos vulnerables de la sociedad en los que se considera a las personas con discapacidad.

Asimismo, esta invisibilización del tema en la Educación Superior puede relacionarse con una escasa articulación y coordinación entre la Administración Nacional de Educación Pública y la Universidad de la República.

Como ya fue señalado si bien se evidencia la construcción de un discurso pedagógico que privilegia la noción de inclusión educativa en la formulación de las políticas educativas nacionales, aún se está lejos de poder consolidar un sistema nacional educativo inclusivo.

Una política de inclusión educativa implica un proceso interminable de transformación del sistema educativo en su conjunto. Para ello es necesario generar procedimientos más eficientes, orientados a identificar y dar respuesta a los estudiantes con discapacidad que egresan del subsistema de la Educación Media y que pretenden continuar sus estudios en la Educación Superior. Esto también plantea otro desafío que es garantizar la permanencia y egreso de estos estudiantes en la Universidad. Sin lugar a dudas, lo anterior demanda a la institución universitaria, por un lado, la erogación de recursos económicos y humanos para brindar a los estudiantes con discapacidad los apoyos necesarios para facilitar el tránsito formativo. Y por otro, sensibilizar a la comunidad universitaria para promover y desarrollar una ética de la hospitalidad (Almeida \& Angelino, 2014).

\section{Referencias}

Almeida, M.; Angelino, M. (2014). De la inclusión educativa como política a la ética de la hospitalidad. Notas para el debate. Psicología, Conocimiento y Sociedad, 4(2), 10 - 27. Recuperado: 3 mar. 2017. Disponível: http://revista.psico.edu.uy/

Arocena, R. (2008). Entrevista a Rodrigo Arocena, rector de la 
Universidad de la República. En Diálogo - Otra puerta se abre, $O(1)$.

Banco Mundial. (2004). Fondo de Inclusión Escolar: La experiencia de Uruguay. Recuperado: 3 mar. 2017. Disponível: http://pdi. cnotinfor.pt/recursos/Fondo\%20de\%20Inclusion \%20Escolar, $\% 20$ Espanol.doc.

Bralich, J. (1994). Historia de la Universidad. Montevideo: Multiplicidades.

Echeita, G. (2006). Educación para la inclusión o la educación sin exclusiones. Madrid: Narcea.

Educación Superior Virtual Inclusiva - América Latina [ESVI-AL] (2013). Informe descriptivo de análisis de accesibilidad en educación superior para personas con discapacidad. Montevideo. Recuperado: 3 mar. 2017. Disponível: http://www.esvial.org/wpcontent/files/E111_vf_v1.pdf

Farías, N.; Viera, A. (2013). Accesibilidad e inclusión educativa en la Universidad de la República. In: Pérez, L.; Fernández, A.; Katz, S. (Orgs.), Discapacidad en América Latina: voces y experiencias universitarias (pp. 261-270). Universidad Nacional de La Plata.

Fernández, T. (2009). Desigualdad, democratización y pedagogías en el acceso a la educación superior de Uruguay. Revista de la educación superior, 38 (152), 13-32. Recuperado: 3 mar. 2017. Disponível: $\quad$ http://www.scielo.org.mx/scielo.php?script=sci_ arttext\&pid=S0185-27602009000400002\&lng=es\&nrm=iso. ISSN 0185-2760.

Ley No. 18.437 de 12 de diciembre de 2008. (2008). Ley General de Educación. Registro Nacional de Leyes y Decretos.

Ley No. 18.651 (2010). Ley de protección integral a las personas con discapacidad. Registro Nacional de Leyes y Decretos.

Lemez, R. (2005). La integración de las personas con discapacidad en la educación superior en el Uruguay. Montevideo: IESALC/ UNESCO.
Mancebo, M.E.; Goyeneche, G. (2010). Las políticas de la inclusión educativa: Entre la exclusión social y la innovación pedagógica. Trabajo presentado en las 9 Jornadas de la Facultad de Ciencias Sociales de la Universidad de la República, Montevideo, Uruguay. Recuperado: 5 mar. 2017. Dlsponível: http://www.fcs.edu.uy/ archivos/Mesa_12_y_17_Mancebo-Goyeneche.pdf

Martinis, P. (2016). Aproximación a los usos del significante "inclusión educativa" en la formulación de políticas educativas en Uruguay (2005-2015). In: Martignoni, L.; Zelaya, M. (Orgs.), Diálogos entre Argentina, Brasil y Uruguay. Sujetos, políticas y organizaciones en educación (pp. 1-15). Buenos Aires: Biblos.

Meresman, S. (2012). La situación de niños, niñas y adolescentes con discapacidad en Uruguay. La oportunidad de la inclusión. Fondo de las Naciones Unidas para la Infancia y Instituto Interamericano sobre Discapacidad y Desarrollo Inclusivo. Montevideo: Masterfraf.

Tejeda, P. (2012). Aproximación a las políticas de educación superior para estudiantes con discapacidad en los países del cono sur: Argentina, Chile y Uruguay. Tesis de Maestría. Universidad Nacional de Colombia, Facultad de Medicina.

Universidad de la República (2007). Hacia la reforma universitaria $n^{\circ}$ 1. Universidad de la República, Montevideo.

Universidad de la República (2008). Creación de la Red Temática sobre Discapacidad de la UDELAR.

Viera. A.; Zeballos, Y. (2014). Inclusión educativa en Uruguay: Una revisión posible. Psicología, Conocimiento y Sociedad, 4(2), 237260. Recuperado: 5 abr. 2017. Disponível: http://revista.psico.edu. uy/

"Pesquisa em Rede "Acessibilidade no Ensino Superior", financiada pelo Programa Observatório da Educação OBEDUC/CAPES - Proc. no 23038.002628/2013-41"

License information: This is an open-access article distributed under the terms of the Creative Commons Attribution License (type CCBY), which permits unrestricted use,distribution and reproduction in any medium, provided the original article is properly cited. 


\section{Sobre as autoras}

Andrea Jimena Gomez (aviera@psico.edu.uy)

Candidata a Doctor (a) en Lingüística por la Facultad de Humanidades y Ciencias de la Educación de la Universidad de la República (FHCEUdelaR). Licenciada en Psicología y Magister en Psicología y Educación por la Facultad de Psicología de la UdelaR. Profesora Adjunta (Dedidación Total) en el Instituto de Psicología, Educación y Desarrollo Humano (IPEDH) de la Facultad de Psicología de la UdelaR. Integra el Sistema Nacional de Investigadores de la Agencia Nacional de Investigación e Innovación de la República Oriental del Uruguay. Es investigadora responsable de dos proyectos de investigación financiados por la CSIC (UdelaR) y de dos Grupos de Investigación Autoidentificados.

https://orcid.org/0000-0002-8827-8781

Yliana Zeballos Fernández (ylianaze@psico.edu.uy)

Licenciada en Psicología y Magister en Derechos de Infancia y Políticas Públicas por la Facultad de Psicología de la UdelaR. Asistente en el Instituto de Psicología, Educación y Desarrollo Humano (IPEDH) de la Facultad de Psicología de la UdelaR. Es docente en el Consejo de Formación en Educación (CFE-ANEP) desde 2006. Integra el Sistema Nacional de Investigadores de la Agencia Nacional de Investigación e Innovación de la República Oriental del Uruguay. Participa en la ejecución de diferentes políticas educativas inclusivas en instituciones educativas públicas de la ANEP en el marco de la implementación del Sistema de Protección de Trayectorias Educativas que lleva adelante el Consejo Directivo Central desde 2015. Es integrante del equipo de investigación y extensión en Inclusión social y educativa del IPEDH de la FP de la UdelaR.

https://orcid.org/0000-0002-4175-0161 